\title{
New methods in arthroscopy: preliminary investigations
}

\author{
L FRIZZIERO, ${ }^{1}$ F ZIZZI, ${ }^{1}$ R LEGHISSA,${ }^{2}$ AND A FERRUZZI
}

From the ${ }^{1}$ Servizio di Medicina Interna, Unità Artrite Reumatoide Centro polifunzionale di Artroscopia, Centro Traumatologico Ortopedico, USL n 27, Bologna, Italy; and the ${ }^{2}$ Istituto di Patologia Speciale Medica e Metodologia Clinica II, Scuola di Specializzazione in Reumatologia, Università di Bologna, Italy

SUMmaRY The microhysteroscope, which affords direct in vivo observation of otherwise inaccessible surfaces, can be used to great advantage in arthroscopy. Although conventional arthroscopy can distinguish between 'inflammatory' and 'reactive' (post-traumatic) synovial changes, the microendoscope offers the possibility of more precise differentiation. Synovial membranes, joint cartilage, and menisci were studied at four different magnifications, including microscopic observation of vitally stained cells. Frankly pathological synovia (e.g., rheumatoid arthritis, psoriatic arthritis, ankylosing spondylitis) were compared and distinguished from examples of less florid synovial changes. The fibrocartilaginous structure of the menisci and secondary undulations and tertiary depression of joint cartilage become visible in vivo with the microendoscope; these may well point to early damage to these structures. The authors believe that the three dimensional images at the magnifications provided by this microendoscope go some way towards bridging the gap between the conventional arthroscope, the light microscope, and the scanning electron microscope. This report presents preliminary findings with this new technique.

Key words: synovial membrane, reactive synovitis.

The microhysteroscope, developed in its final form in 1979 by Messrs Karl Storz in collaboration with the French gynaecologist, Hamou, affords direct in vivo observation of otherwise inaccessible surfaces at various magnifications $(10 \times$ to $150 \times)$.

The authors believe that this instrument can be used to great advantage in arthroscopy. This report describes preliminary findings with this technique.

\section{Materials and methods}

The $25 \mathrm{~cm}$ long, $4 \mathrm{~mm}$ Storz microhysteroscope has a field of $90^{\circ}$ and a $30^{\circ}$ foreoblique vision. Light is provided by a cold $150 \mathrm{~W}$ source, with transmission by a flexible optic fibre cable. Cameras for photography, cinematography, or TV can be attached. The unique feature of the microhysteroscope is the ability to pass from panoramic vision to contact view

Accepted for publication 5 November 1985.

Correspondence to Dr L Frizzicro, Servizio di Medicina Interna, CTO via Boldrini 2, Bologna, Italy. at four different magnifications, including microscopic observations of vitally stained cells. ${ }^{2}$ The different magnifications are indicated on a four position index on the ocular.

After conventional arthroscopy the joint cavity was carefully washed with saline to which $3 \mathrm{ml} 1 \%$ methylene blue was added in the final stages. Endoscopic exploration was then commenced at increasing magnifications. Informed consent was obtained from each patient.

Unit magnification (position 1) afforded a preliminary panoramic view of the joint cavity; more restricted but detailed panoramic vision was obtained with $30 \times$ magnification (position 2). At $60 \times$ magnification (depth of field approx. $80 \mathrm{~nm}$ ) the synovial and villous architecture was visible as well as the blood vessels and the passage of red blood corpuscles. A magnification of $150 \times$ provided contact microscopic observation of superficial cells; focusing was automatic. Blood and synovial fluid did not hamper, but rather enhanced vision, being immersion media with high refractive indices. 
Furthermore, the high resolution of the microhysteroscope made it comparable with a normal $150 \times$ magnification microscope. ${ }^{1}$

\section{Results}

SYNOVIAL MEMBRANE

Normal arthroscopy can distinguish between inflammatory' and 'reactive' (post-traumatic) synovium. The microendoscope can provide more detailed information. Correct interpretation, however, depends on extensive familiarity with the various, often misleading, patterns of the synovial villi as reported by Watanabe in $1969 .{ }^{3}$

With panoramic vision the synovial membrane of the suprapatellar pouch appeared thin, smooth, glossy, transparent, and outlined by the subintimal and subsynovial vascular plexus with its fine, tortuous favus like pattern (Fig. 1). Even in the young subject short, thin, translucent villi were seen on the femoral surface close to the chondrosynovial junction, with blood vessels clearly visible.

At $30 \times$ magnification the smooth appearance seen at unit magnification now presented slight ridges or undulations. There was better resolution of the complex vascular network in the stroma, from whence the capillary loops supplying the synovial villi arise. ${ }^{4}$ These capillary vessels of the synovial membrane undergo progressive transformation with advancing age: the young person presents an extensive intricate network of vessels with long thin capillary loops lying close to the surface; in the elderly, vessels appear further from the synovial surface and the loops are wire like, shrunken, and rigid.

At $60 \times$ and especially at $150 \times$ magnifications the



Fig. 1 Normal synovial membrane: thin translucent villi; normal cellularity of synovial lining. The subsynovial vascular plexus is visible with its fine, tortuous honeycomb pattern. In the background adipose tissue can be seen. individual synoviocytes making up the synovial intima were clearly seen. These magnifications were $\frac{\square}{\omega}$ employed to compare frankly pathological synovia with others regarded as normal or only slightly $\underset{\sim}{\vec{S}}$ abnormal ('reactive').

The intimal cells of normal synovial membranes, and more especially 'reactive' synovia after recent trauma, appeared star shaped with long prolongations (Fig. 2). (Ultramicroscopic studies show the predominance of A synoviocytes under these conditions. ${ }^{5}$ ) Despite high cellularity the villi still appeared transparent (Figs 3 and 4).

Rheumatoid joints characteristically showed $\stackrel{\omega}{\mathscr{\omega}}$ chronic proliferative synovitis. Very advanced villous hyperplasia is associated with turgid, hyperaemic, ground glass type tissue.

Greater magnification showed hypercellularity, iे predominantly of round cells against an opaque, ill

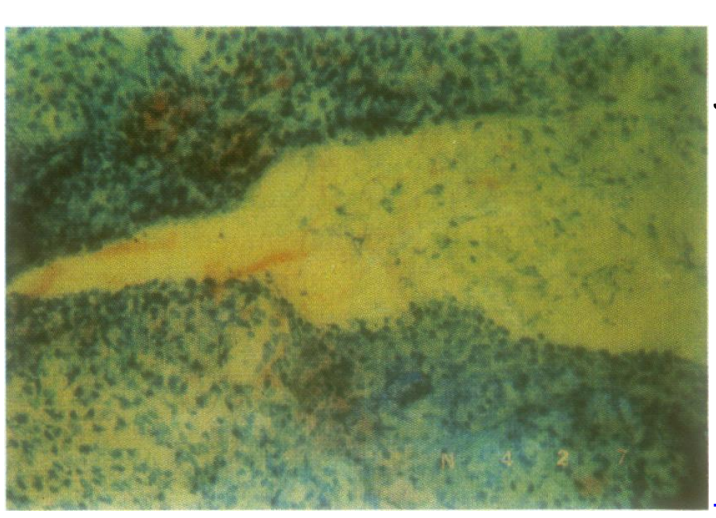

Fig. 2 Reactive synovitis: the synoviocytes are numerous and appear star shaped with clear outlines. In the middle, adipose tissue with reduced colouring can be seen.

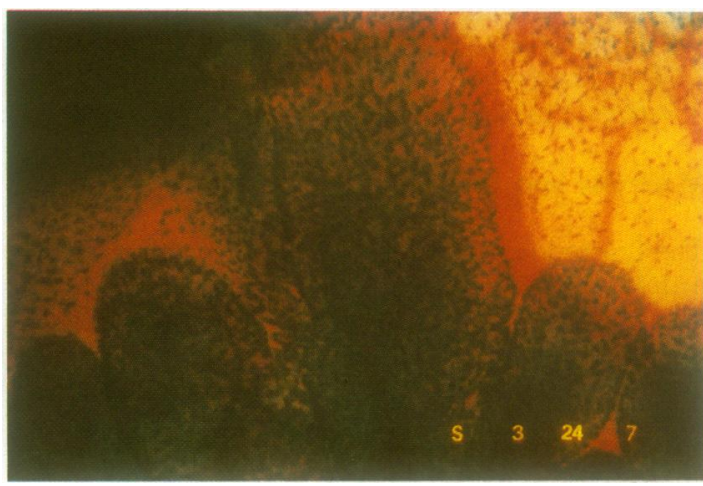

Fig. 3 Reactive synovitis: translucent villi with star shaped hypercellularity; the synovial lining is preserved with normal cellularity. The vascular network is visible. 


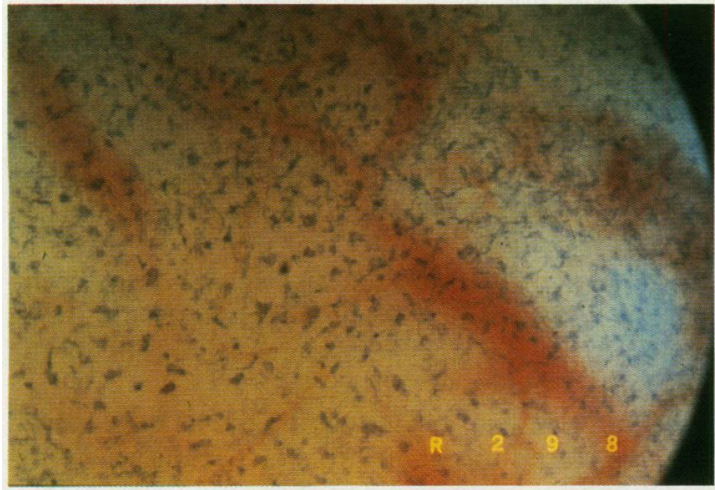

Fig. 4 Reactive synovitis: translucent hyperaemic villi with clear vascular network. There are more star shaped synoviocytes than in normal joints but fewer than in rheumatoid arthritis.

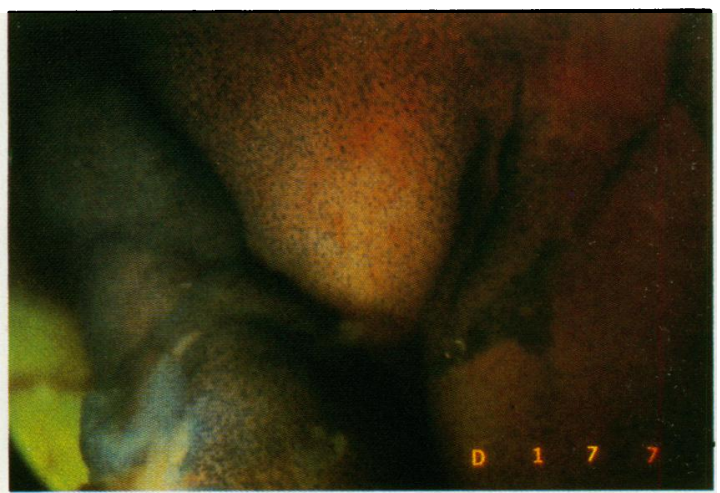

Fig. 5 Rheumatoid synovitis: hypertrophic, rounded, polyp like villi with an opaque, ill defined background due to congestion and oedema. Mixed hypercellularity, mainly of rounded cells; the vascular network is confused, irregular.

defined background due to congestion and oedema. These were probably B type synoviocytes which are known to increase in the intima with the onset of rheumatoid arthritis (Fig. 5).

The vascular plexus often showed marked changes. Marin et al maintain that these morphological and functional alterations originate in the deep vascular layer and are secondary to vasculitis ${ }^{\mathrm{b}}$ : the microvascular circulation breaks down; the venules are swollen and irregular with turgid flow and extravasation of blood (Fig. 6) ${ }^{7-9}$ Hypertrophy of the intima is described with extravasated erythrocytes secondary to microthrombi and vasculitis. ${ }^{610-12}$

The changes in the surface cells may have resulted

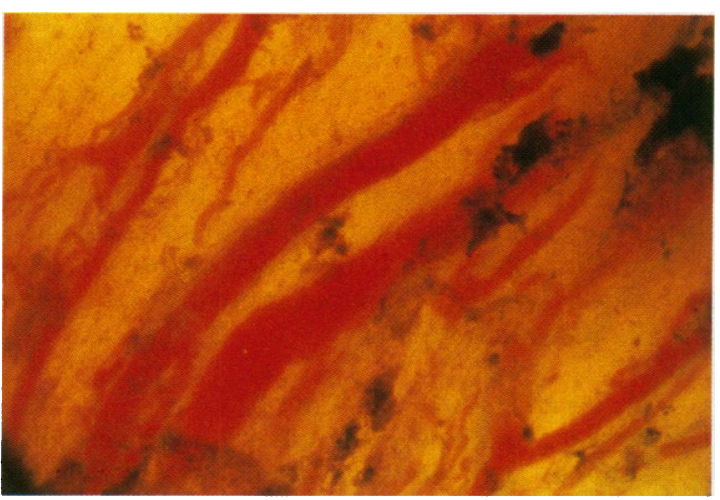

Fig. 6 Reactive synovitis: the vascular network is clearly visible with dilated and straight vessels.

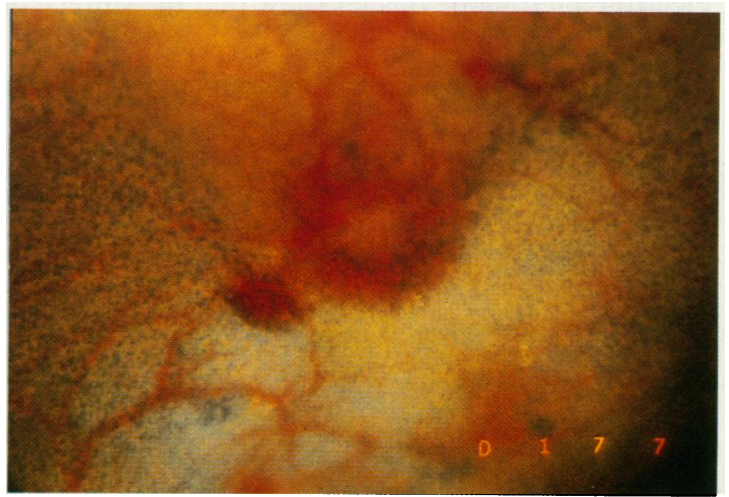

Fig. 7 Rheumatoid synovitis: the vascular network is irregular with no clear outlines; there is an extravasation of blood with a pale central part ringed by a dark red area.

from the failure of the intercellular circulation and the formation of antigen-antibody complexes. ${ }^{6}$

Microarthroscopy showed a sparse irregular vascular plexus with only a few truncated ramifications, probably the result of exudate and cell infiltrate compressing the microvessels. Sometimes clusters of micropetechiae were observed on the suprapatellar pouch with panoramic vision. At higher magnifications each such cluster showed a pale central part (the vessels occluded by the microthrombus or vasculitis) ringed by a dark red area (extravasated blood) (Fig. 7)

Reactive or mechanical synovitis may also produce petechiae but of different appearance: more turgid and drop like in form, indicating the essential haemorrhagic nature of the condition due to broken vessel walls. ${ }^{11}$ 
The microendoscopic appearance of synovitis due to psoriatic arthritis and ankylosing spondylitis was characterised by slight to moderate hypertrophy of synoviocytes and by villi which were less thin, more translucent, and less pedunculated and ramified than those in rheumatoid synovitis. In this respect the microscopic appearance of synovitis secondary to psoriatic arthritis and ankylosing spondylitis was half way between rheumatoid synovitis and mechanically induced synovitis.

\section{JOINTCARTILAGE AND MENISCI}

The cartilage of the patellofemoral joint can usefully be studied by arthroscopy, which allows observation of damage and also possibly insight into pathogenetic mechanisms (Fig. 8). Microarthroscopy allows observation in vivo of features that ultramicroscopy has recently begun to provide in vitro. Scanning electron microscope images show that the surface of

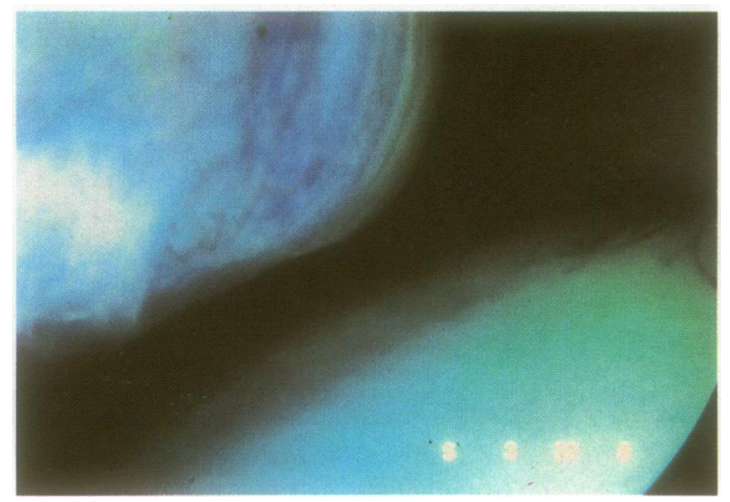

Fig. 8 Normal patellofemoral joint: the surface of the articular cartilage is smooth, glossy with fine, parallel undulations.

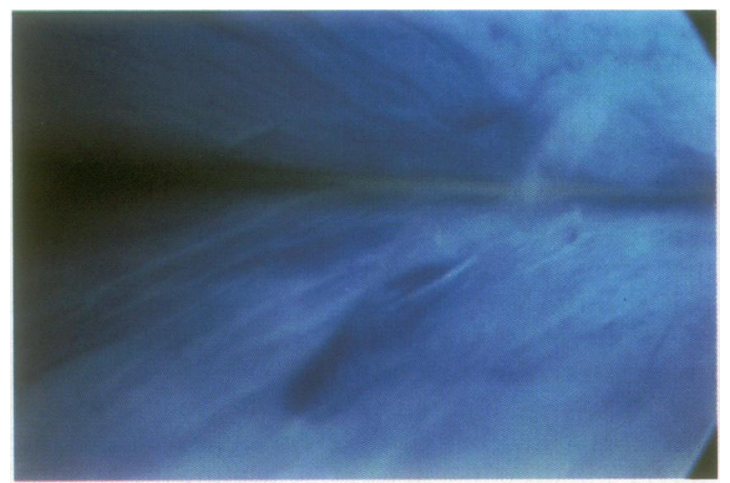

Fig. 9 Patellofemoral joint: the parallel undulations (second class) lie along the plane of movement. cartilage is not, as was once thought, smooth and homogeneous, but exhibits four stages of increasing irregularity and undulations with advancing age. ${ }^{4}$

Panoramic arthroscopy showed the primary outline clearly. With the microendoscope second class undulations $(200-400 \mathrm{~nm})$ became visible; tertiary depressions $(15-45 \mathrm{~nm} \times 0 \cdot 6-3 \mathrm{~nm})$ were less easy to identify (Fig. 9).

In the femoral trochlea the sulci lie along the plane of movement. The undulations on the tibial plate are perpendicular to the free border of the meniscus.

At intermediate magnification the healthy meniscus apppeared turgid and compact, indicative of its function as a protective pad (Fig. 10). 150 $\times$ magnification showed clearly the fibrocartilaginous structure of the meniscus, composed of coarse collagen bundles very similar to the fibrous connective tissue of tendons and the joint capsule itself (Fig. 11).

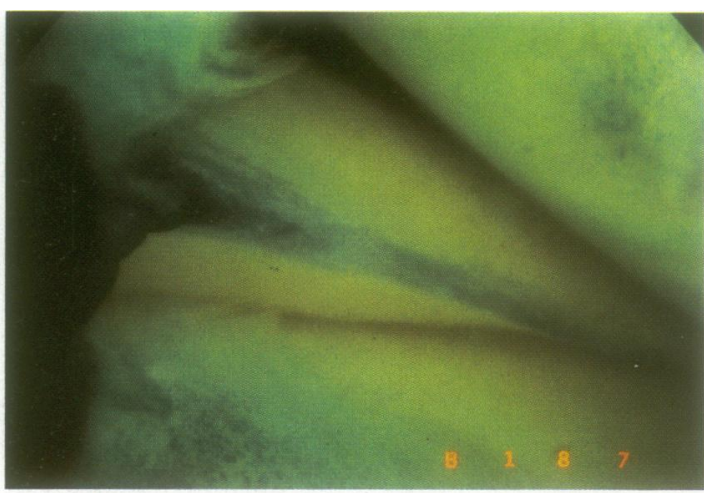

Fig. 10 Normal medial meniscus: the middle segment is turgid and compact, indicative of its function.

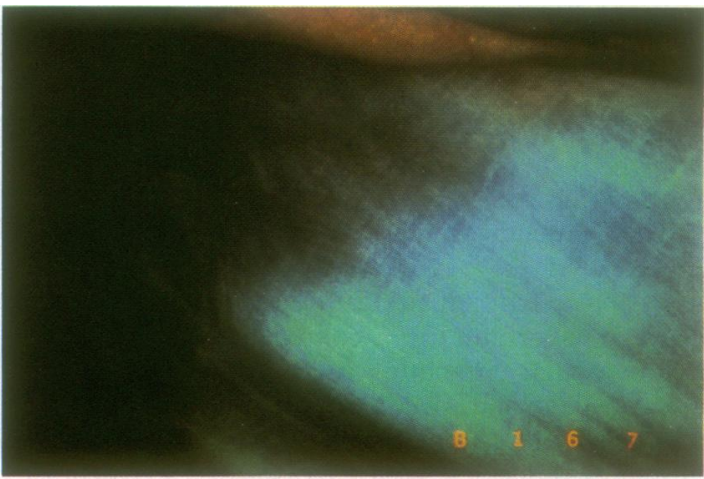

Fig. 11 Meniscus: fibrocartilaginous structure of the meniscus composed of coarse collagen bundles. 


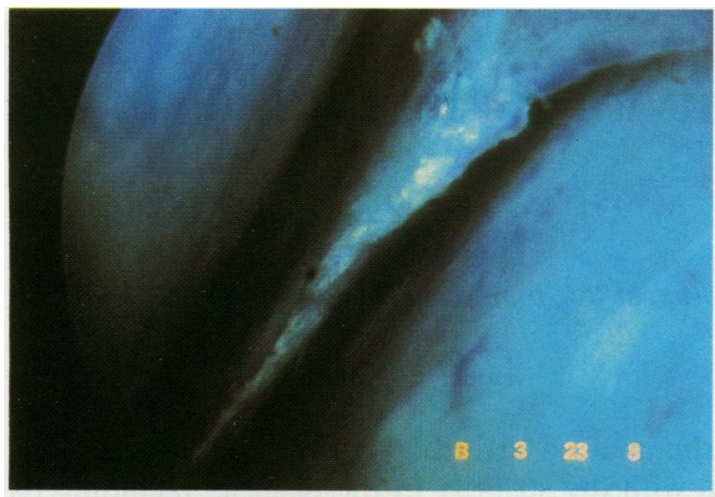

Fig. 12 Lateral meniscus: calcium pyrophosphate crystals on the inner margin.

These bundles of collagen fibres do not lie parallel but intertwine in various planes, thereby probably conferring mechanical resistance. Altered staining patterns, irregular or anomalous outline of the surface cartilage or meniscus, or both, could be identified with microendoscopic vision and may well point to early damage of these structures (Fig. 12).

\section{Discussion}

Care must be exercised when interpreting these findings, given the limited experience of the authors, but the instrument appears to have great potential for use in both research and diagnosis.

The three dimensional images at the different magnifications provided by this microendoscope begin to bridge the gap between the conventional arthroscope, the light microscope, and the scanning electron microscope, with the further advantage that the observations are made in vivo.

The authors believe that many novel, previously uncontemplated applications are open to the microendoscope, not only in orthopaedics but also in rheumatology. Operator skill and experience in using the instrument and interpreting the images is essential for the effective usefulness of the microendoscope to be realised. ${ }^{2}$

\section{References}

1 Hamou J E. Microhysteroscopie une nouvelle technique en endoscopie, ses applications. Acta Endoscopica 1980; 10: 415-22.

2 Hamou J E. Microhysteroscopy-a new procedure and its original applications in gynecology. J Reprod Med 1981; 26: $375-82$.

3 Watanabe M, Takeda S, Ikeuchi H. "Atlas of arthroscopy. 2nd ed. Tokyo: Igaku Shoin Ltd, 1969.

4 Fantini F, Tosi S, Rizzi S. Morfologia reumatologica a scansione. Milan: Farmitalia, 1978.

5 Letizia G, Nesci E, Piccione F, Ridola C V, Valentino B, Zummo G. Struttura ed ultrastruttura della membrana sinoviale. LXIII Congresso SIOT. Palermo, 23-26 Novembre 1978. (GIOT suppl pp. 19-30.)

6 Marin D, Negoescu M. Stoia I. Pierrette A. Petrescu A L. Constantinescu S P. The morphology of the synovial tissue and articular fluid cells in rheumatoid polyarthritis, studied with the optical and electronic microscope. Acta Rheumatol Scand 1969; 15: $126-31$.

7 Branemark P I. Ekholm R, Goldie J, Lindström J. Synovectomy in rheumatoid arthritis. Experimental biological and clinical aspects. Acta Rheumatol Scand 1967; 13: 161-7.

8 Golide I. The synovial microvascular derangement in rheumatoid arthritis and osteoarthritis. Acta Orthop Scand 1970; 40: 751-6.

9 Jalava $S$. Irradiation synovectomy in experimental arthritis and in human and rhcumatoid arthritis. Scand J Rheumatol 1974; 2 (suppl 4): 1-32.

10 Soren A, Klein W. Huth F. The synovial changes in posttraumatic synovitis and ostcoarthritis. Rheumatol Rehabil 1978; 17: $38-45$.

11 Soren A. The diagnostic significance of certain histopathologic changes for rheumatoid arthritis. $Z$ Rheumatol 1980; 39: 74-8.

12 Revell P A. Mayston V. Histopathology of the synovial membrane of peripheral joints in ankylosing spondylitis. Ann Rheum Dis 1982; 41: 579-86.

13 Ficat P. Cartilage et arthrose. Exploration functionelle pathologie et therapeutique. Paris: Masson, 1978. 